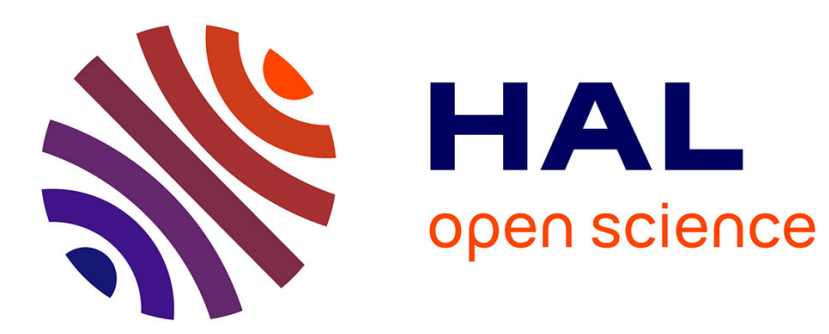

\title{
Thermoresistance and Giant Magnetoresistance of Magnetorheological Elastomers
}

\author{
Nadir Kchit, Pascal Lancon, Georges Bossis
}

\section{To cite this version:}

Nadir Kchit, Pascal Lancon, Georges Bossis. Thermoresistance and Giant Magnetoresistance of Magnetorheological Elastomers. Journal of Physics D: Applied Physics, 2009, 42, pp.105506. 10.1088/0022-3727/42/10/105506 . hal-00455576

\section{HAL Id: hal-00455576 https://hal.science/hal-00455576}

Submitted on 10 Feb 2010

HAL is a multi-disciplinary open access archive for the deposit and dissemination of scientific research documents, whether they are published or not. The documents may come from teaching and research institutions in France or abroad, or from public or private research centers.
L'archive ouverte pluridisciplinaire HAL, est destinée au dépôt et à la diffusion de documents scientifiques de niveau recherche, publiés ou non, émanant des établissements d'enseignement et de recherche français ou étrangers, des laboratoires publics ou privés. 


\title{
Thermoresistance and Giant Magnetoresistance of Magnetorheological Elastomers
}

\author{
N Kchit, P Lancon and G Bossis \\ L.P.M.C. UMR-CNRS 6622, University of Nice Sophia Antipolis, 06108 Nice cedex 2, \\ France. \\ E-mail: bossis@unice.fr
}

\begin{abstract}
.
A new magnetorheological elastomer composite (MRE) was developed with a resistance which is strongly sensitive both to temperature and pressure. The electrical resistance of this MRE was investigated versus pressure and temperature. The resistance of the composite was recorded versus pressure for seven different values of the temperature. The mechanism of electrical conduction was modeled and assessed experimentally. An other type of composite, prepared with a softer elastomer, showed a giant magnetoresistance with a decrease by 3 orders of magnitudes for a field of only $100 \mathrm{kA} / \mathrm{m}$ (1250Oe); It was shown that the magnetic pressure acted like mechanical pressure for field lower than $100 \mathrm{kA} / \mathrm{m}$ but beyond the magnetoresistance was saturating contrary to piezoresistance.
\end{abstract}

\section{Introduction}

The electrical conductivity of conductive polymer composites (CPC) including metal powder or carbon black fillers has been widely studied as a function of both filler concentration and external stimulus. The CPC prepared slightly above the percolation threshold exhibit a large change in resistivity with different stimuli that induce a change in the arrangement of conductive filler particles. The control of the interparticle separation is at the origin of applications like temperature $[1,2,3]$, pressure [4] and chemical sensors $[5,6]$.

This paperis devoted to the study of thermoresistance and magnetoresisrance of so called magnetorheological elastomer MRE which consists in a structured dispersion of micron size magnetic particles inside an elastomer matrix. During the curing, the suspension is subjected to a strong magnetic field that creates an attractive interparticle force parallel to the field direction, thus arranging the initially homogeneous dispersion in a chain-like structure [7]. The main advantages for using this material rather than a conventional CPC is that MRE can be conductor even at low particle concentration and also is more sensitive to pressure than $\mathrm{CPC}[8,9]$. In a previous paper [9], we have shown that the change of electrical resistivity with pressure of a MRE composite cured at room temperature could be well predicted by a model based on tunnel conduction 
between asperities separated by an oxide layer and a thin compressible polymer layer. In this paper we present experimental results for the piezoresistance of a composite which was cured both under heating at $80^{\circ} \mathrm{C}$ and in the presence of a magnetic field. The experimental piezoresistance recorded at different temperatures was compared to this model which was extended to take into account the effect of temperature on the local strain. In the last section it is shown that, using a softer elastomer as a matrix for the MRE, it was possible to obtain a giant magnetoresistance; this effect is interpreted on the same basis as piezoresistance.

\section{Sample preparation}

Two commercial elastomers were used to make our MRE composites, silicone RTV 141A and RTV 1062A associated respectively to RTV141B and RTV 1062B hardener from Rhodia. The fillers are nickel particles from MERCK kgaA with mean diameter $10 \mu \mathrm{m}$. The roughness of this fillers was characterized with the help of AFM technology.

The preparation of the composites with the application of a magnetic field before the completion of polymerization was already described $[7,11]$ the only addition here is the presence of a heating filament around the rotating mold. The time of polymerization can take over 24 hours at room temperature but less than one hour when heating at $80^{\circ} \mathrm{C}$ with the polymer RTV 141 . For the sample used for magnetoresistance the filler particles are the same but the polymer is the RTV 1062 and the curing was made only at room temperature, since higher temperatures inhibit the crosslinking of these molecules. The sample is a flat cylinder with $20 \mathrm{~mm}$ of diameter and $2 \mathrm{~mm}$ in height.

\section{Results and discussion}

The composite MRE Ni-Merck 30\% with RTV141 cured at room temperature has still a high resistivity close to $10^{8} \Omega . c m$. In fact, during the polymerisation of the composite, the particles come only slightly in contact under the influence of the magnetic field and the high value of resistivity after curing at $80^{\circ} \mathrm{C}$ suggests that a thin layer of polymer remains on the surface of the particles. This composite exhibits a large piezoresistance due to the variation of the thickness of the adsorbed polymer layer and of the contact area between asperities under pressure [9]. When the sample is cooled from $80^{\circ} \mathrm{C}$ to room temperature, the resistance strongly decreases to reach a value as low as $10 \Omega . c m$. This is due to the fact that the thermal expansion coefficient of the polymer is an order of magnitude larger than the one of the nickel particles, so the polymer shrinks more during cooling than the nickel particles. This polymer shrinkage induces a compressive force which is still more efficient than the magnetic field to bring particles into contact, explaining the low resistivity of composite at room temperature[10, 11]. This type of composite has very interesting properties for thermoresistance applications.

The composite MRE Ni-Merck $30 \%$ with RTV141 prepared at $80^{\circ} \mathrm{C}$ was inserted into the thermal chamber between the two plates of a dynamic mechanical thermal 
analyser DMA +450 from Metravib. The sample was heated to $80^{\circ} \mathrm{C}$ and then cooled by steps of $10^{\circ} \mathrm{C}(70,60,50,40,30$ and ambient temperature); for each level of temperature the sample undergoes a cycle of compression-decompression. The results are presented in Fig (1).

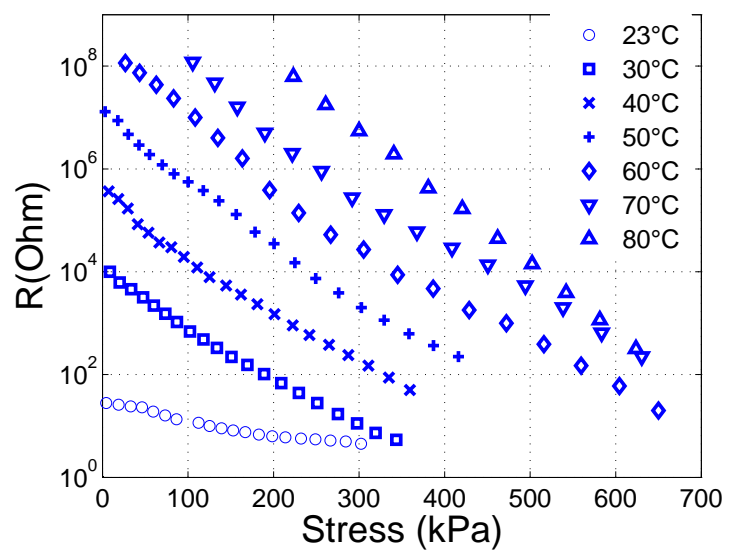

Figure 1. Resistance versus applied pressure for different temperatures

At $80^{\circ} \mathrm{C}$, a finite value of resistance is obtained only at high value of compression: $210 \mathrm{kPa}$ and then with cooling the sample required less and less stress to start measuring resistance. This is explained by the larger thickness of the polymer layer which remains between the asperities at high temperature. As the composite is cooled, the layer thickness decreases due to the compressive force of shrinkage. This decrease of thickness will be highlighted through the model summarized below [9].

The total resistivity of composite is based on tunnel conduction through small contact area between asperities:

$$
\rho_{c}=\frac{\pi}{6} \frac{d}{\phi} \frac{\rho_{t}}{A_{c}}
$$

$d$ is the mean diameter of particles, $\phi$ is the volume fraction of particles, $A_{c}$ is the real contact area which is given versus the macroscopic strain $\epsilon$ by [9]:

$$
A_{c}(\epsilon)=2 \pi r_{a} N_{a} \int_{0}^{\infty} x \exp \left[-\frac{\left(x+h_{T}\left(1-\frac{d}{h_{T}} \epsilon\right)-h_{m}\right)^{2}}{2 \sigma^{2}}\right] d x
$$

and $\rho_{t}$ is the tunnel resistance:

$$
\rho_{t}=\rho_{t}\left(g_{0}\right) \frac{g_{0}+t}{g_{0}} \exp (\gamma t)
$$

with $\gamma=1.024 \sqrt{\varphi}$ expressed in $(1 / \AA)$, where $\varphi$ is the height of potential barrier equal to $0.7 \mathrm{ev}, t$ and $g_{0}$ are respectively the thickness of polymer layer and oxide layer, $h_{T}$ is the initial separation at zero pressure between the average surfaces of two neighbour particles and $N_{a}, r_{a}, \sigma$ and $h_{m}$ are the roughness parameters measured by AFM: $N_{a}=30$ : 
is the number of asperities which can enter into contact between two particles, $r_{a}=100 \mathrm{~nm}$ is the average radius of curvature of asperities, $\sigma=90 \mathrm{~nm}$ the quadratic mean of the height distribution of asperities and $h_{m}=500 \mathrm{~nm}$ is half of the maximum between peak and dip of the surface. In order to predict the change of resistance with temperature or with strain we need to express the initial gap, $h_{T}$, between two particles versus temperature and the change of thickness of the polymer layer versus temperature or equivalently macroscopic strain.

\subsection{Initial separation $h_{T}$}

At $80^{\circ} \mathrm{C}$ the initial separation at zero pressure between the two mean surfaces, noted $h_{80}$, is supposed to be two times $h_{m}$ since the particles are just brought in contact by the magnetic forces. The initial separation $h_{T}$ at lower temperatures is calculated with the help of the thermal dilatation coefficient $\alpha=2 \cdot 10^{-4} C^{-1}$ of the polymer. Neglecting the dilatation of the bulk nickel we shall have for the thermal strain: $\epsilon=\alpha \Delta T$ with :

$$
\epsilon=\frac{\delta(h+d)}{h+d} \cong \frac{\delta h}{d}
$$

then

$$
h_{T}=h_{80}-\alpha d(80-T)
$$

The values of $h_{T}$ are reported in Table(1)

\begin{tabular}{c|c|c|c|c|c|c|c}
\hline $\mathrm{T}^{\circ} \mathrm{C}$ & 80 & 70 & 60 & 50 & 40 & 30 & 23 \\
\hline$h_{T}(\mathrm{~nm})$ & 1000 & 980 & 960 & 940 & 920 & 900 & 886 \\
\hline
\end{tabular}

Table 1. Initial gap $h_{T}$ between the two mean surfaces at different temperatures

\subsection{Thickness of polymer layer}

The change of thickness of the polymer layer was shown to be given by :

$$
t=t_{T}\left(1-\epsilon_{g}\right)
$$

where $t_{T}$ is the initial thickness of the polymer layer at zero pressure which depends on temperature, $\epsilon_{g}$ is the strain of the polymer layer related to the macroscopic strain by [9]:

$$
\epsilon_{g}=\frac{2}{3 \phi} \frac{h}{h_{T}} \frac{E}{E_{g}} \epsilon
$$

with

$$
\frac{h}{h_{T}}=\left(1-\frac{d}{h_{T}} \epsilon\right)
$$


where $E$ the modulus of composite given by the experimental stress-strain curve of composite Fig(2) and $E_{g}$ is the modulus of the polymer without any filler. Using $\operatorname{Eq}(6)$ the change of thickness $t$ is:

$$
t=t_{T}\left[1-2\left(1-\frac{d}{h_{T}} \epsilon\right) \frac{E}{E_{g}} \epsilon\right]
$$

Note that in this equation $\epsilon$ can be either an imposed external strain or a thermal strain

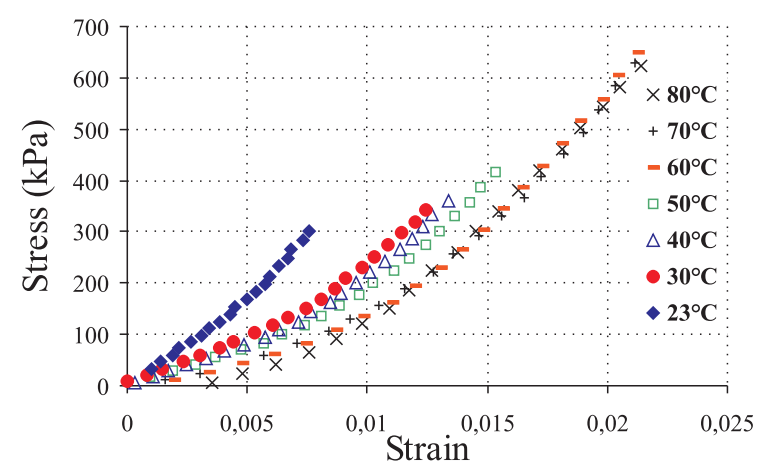

Figure 2. Stress-strain curve at different temperatures

\begin{tabular}{c|c|c|c|c|c|c}
\hline $\mathrm{T}^{\circ} \mathrm{C}$ & 80 & 70 & 60 & 50 & 40 & 30 \\
\hline$t_{T}(\AA)$ & 24 & 18 & 15 & 12 & 8 & 5 \\
$a \times 10^{6}$ & 1 & 1.4 & 1.28 & 1.17 & 0.4 & 0.4 \\
$b$ & 0.98 & 1.1 & 1.02 & 0.92 & 0.57 & 0.5 \\
\hline
\end{tabular}

Table 2. Initial thickness, $t_{T}$, of the adsorbed polymer layer and parameters a,b for the fit of the Young modulus, E, of the composite versus the strain

In order to compare the experimental results to the predictions of the model we need to plot the resistance versus the strain, which is recorded at the same time as the stress. We also need to introduce the modulus of the polymer $E_{g}=500 \mathrm{kPa}$ and to fit the stress strain curves of the composite in order to obtain the dependence of the Young modulus, E, on the strain in $\mathrm{Eq}(7)$. The fit was done with the expression $\mathrm{E}(\epsilon)=\mathrm{a} \epsilon^{b}$. The values of the coefficient $a$ and $b$ are reported in the table (2). The only remaining parameter, that we have used to fit the experimental curves, is the initial thickness of the polymer layer at different temperatures $t_{T}$. As can be seen in Fig (3) the result of the fit is excellent for all the temperatures.

The model can now be used to predict the thermo resistance at any temperature between $80^{\circ} \mathrm{C}$ and $20^{\circ} \mathrm{C}$ and for any strain between 0 and 0.02 . For example we have drawn in Fig (4) the change of resistance with temperature at zero strain. The model, represented by the solid line, allows to predict a resistance of about $10^{14} \mathrm{Ohm}$ at $80^{\circ} \mathrm{C}$ that we were not able to measure with our device. 


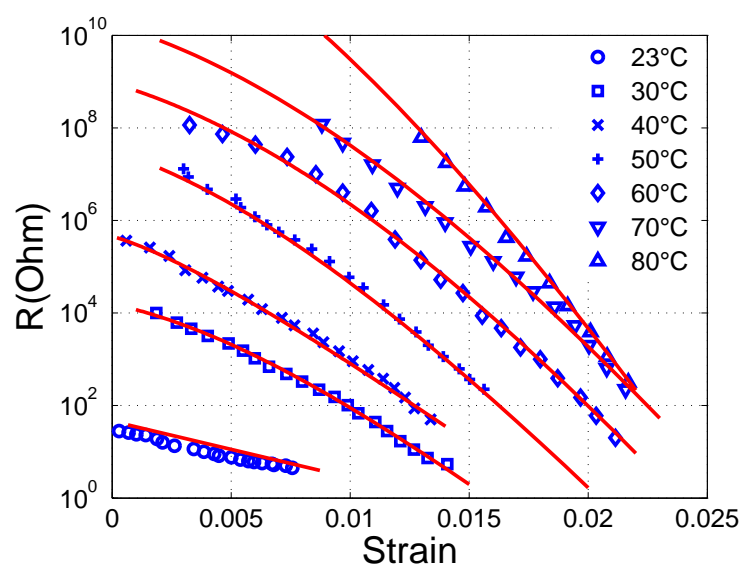

Figure 3. Resistance versus strain; the solid lines are the predictions of the model $\mathrm{Eq}(1)$

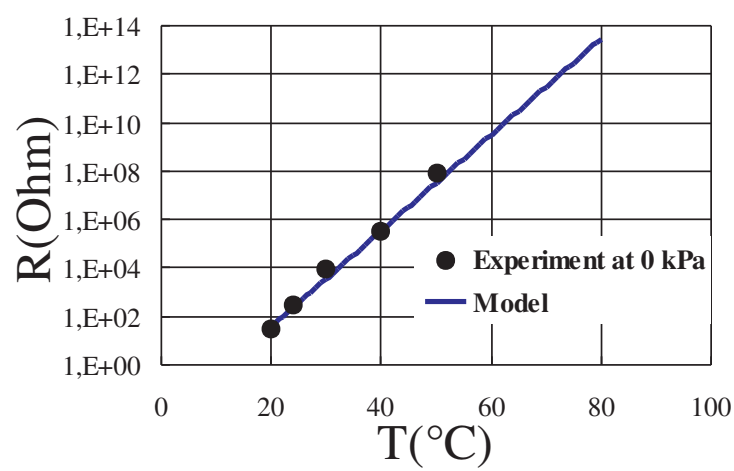

Figure 4. Resistance versus temperature of cooling; the solid lines are the predictions of the model $\mathrm{Eq}(1)$

\subsection{Magnetoresistance}

The MRE composite used to study the magnetoresistance was made using the polymer RTV1062 because its elastic modulus $E_{g}=200 \mathrm{kPa}$ is lower than the one of RTV141 and then allows larger displacement of the particles inside the polymer matrix, still keeping a low restoring time after turning off the field. Two MRE composites of volume fraction $10 \%$ and $30 \%$ were prepared.It was difficult to incorporate more than $10 \%$ of particles in volume so, in order to incorporate $30 \%$, of particles we have reduced the initial viscosity of the RTV1062 by adding silicon oil.After curing under magnetic field, the samples were rectangular plates with dimension $50 \times 30 \times 2 \mathrm{~mm}$. When measuring the resistance, the magnetic field was applied parallel to the direction of the chains, that is to say parallel to the shorter dimension of the plate, and the sample was placed just at equal distance from the poles of the electromagnet in order to avoid the presence of a magnetic force induced by a field gradient.

Fig (5) shows the variation of the relative electrical resistance versus applied 
magnetic field. The Magnetoresistance (MR) is more important in the case of composite at volume fraction $30 \%$ than for the volume fraction of $10 \%$. At $200 \mathrm{kA} / \mathrm{m}$ the resistivity decrease is about $2 \times 10^{4}$ which is a very strong effect compared to usual values even compared to what is called giant magnetoresistance. Actually this decrease of resistance by application of a magnetic field has nothing to do with the spin dependence of electron scattering but with the change of the thickness of the tunnel barrier as we are going to show.

If we believe that the effect of the magnetic field is the same at the one of a mechanical pressure, we have to compare the effect of a mechanical pressure to that of a magnetic pressure. However, in this aim, the applied magnetic field must be converted in terms of a pressure. Using simulation by finite elements (free software FEMM), the magnetic force was calculated between two smooth particles inside chains of particles separated by a distance $2 h_{m}$ and, supposing a chain like structure. The saturation magnetization, $M_{s}$, for nickel material is $490 \mathrm{kA} / \mathrm{m}$ and we have measured on nickel wires an initial permeability $\mu_{i}=54$. The dependence of the permeability versus the field was represented by the Frohlish Kennely formula :

$$
\mu(H)=1+\frac{M_{s}\left(\mu_{i}-1\right)}{M_{s}+\left(\mu_{i}-1\right) H}
$$

The magnetic pressure on the sample is obtained by multiplying the magnetic force between two particles inside a chain by the number of chains per unit surface. The comparison for the change of resistance with mechanical or magnetic pressure is reported in (6). Below $15 \mathrm{kPa}$ corresponding to $100 \mathrm{kA} / \mathrm{m}$, the magnetic pressure acts like a mechanical pressure but above this value the effect of magnetic pressure becomes much lower than the effect of mechanical compression. A possible explanation is that the magnetic force was calculated between two spherical particles with a smooth surface whereas the roughness is about 0.1 times the diameter. The concentration of the field in the asperities can lead to a quicker saturation of the magnetization of the asperities and then to a saturation of the magnetic force at lower fields than for two smooth spheres.

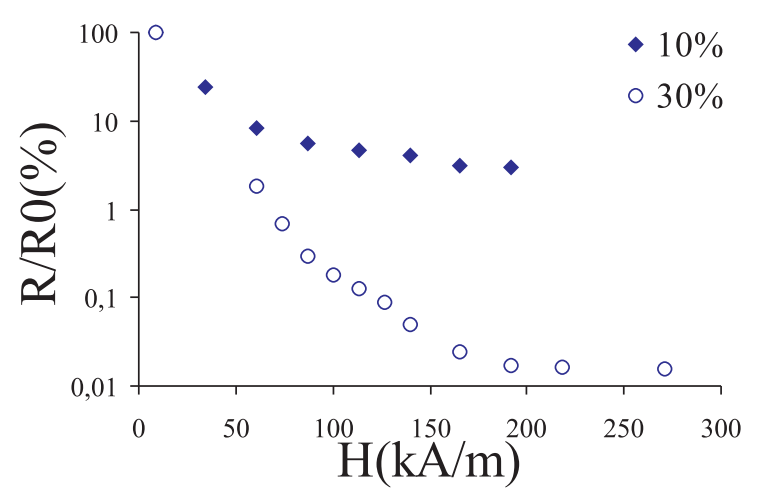

Figure 5. relative decrease of the resistance of two composites at $10 \%$ and $30 \%$ versus the applied magnetic field 


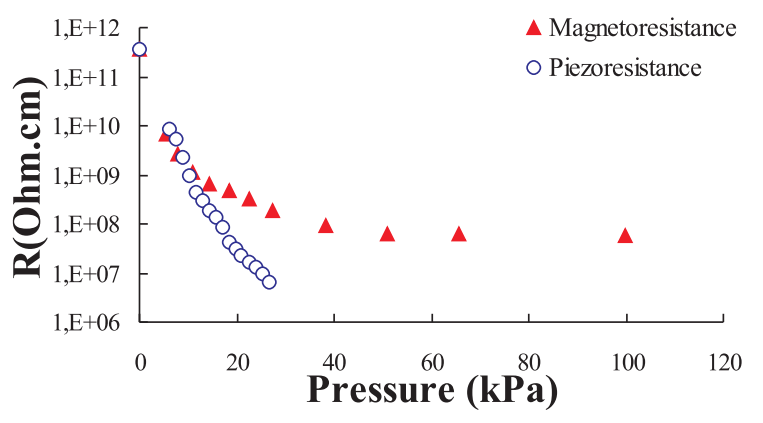

Figure 6. Comparison for the change of resistance due to a mechanical or a magnetic pressure

\section{Conclusion}

We have prepared a new kind of MRE composite by using both heating and field structuring. In this way,during cooling, a strong compressive stress was induced, due to the difference in thermal expansion coefficient between the nickel and the polymer, which gave to the composite a low resistance at room temperature. With this copmposite a large range of resistance values can be attained either by further heating(temperature sensor) or by combining heating and mechanical stress (pressure and temperature sensor). A model previously developed to predict the piezoresistance was improved to include thermal strain and was successfull in predicting both thermoresistance and piezoresistance. Using a softer silicone polymer cured at room temperature, it was possible to change the resistance of the composite by 4 orders of magnitude by applying a moderate magnetic field of $200 \mathrm{kA} / \mathrm{m}$. We have seen that this magnetoresistance was produced by the magnetic attractive force between two particles which has an analogous effect to the application of a mechanical pressure. Several applications, like for instance microconnectors controlled by a local magnetic field, could be developed with this kind of material

\section{References}

[1] Carmona F and Mouney C J 1992 Mater. Sci. 275

[2] Feller J F, Linossier I and Grohens Y 2002 Mat. Lett. 5764

[3] Lundberg B and Sundqvist B 1986 J. Appl. Phys. 601074

[4] Jin S, Mottine J, John J and Sherwood R C 1987 U.S. Patent. 4,644,101

[5] Barkauskas J 1997 Talanta. 441107

[6] Kim Y S, Ha S C, Yang Y, Kim Y J, Cho S M, Yang H and Kim Y T 2005Sens. Actuators. B. 108285

[7] Coquelle E and Bossis G 2005 Journal of Advanced Science 17

[8] Martin J E, Anderson R A, Odinek J, Adolf D and Williamson J 2003 Phys. Rev. 67094207

[9] Kchit N and Bossis G, J. Phys. D: Appl. Phys. submitted

[10] Sancaktar E and Wei Y 1996 J. Adhesion. Sci. Technol. 101221

[11] Kchit N and Bossis G 2008J. Phys.: Condens. Matter 20 